\title{
Edge Gyrokinetic Theory and Continuum Simulations
}

X.Q. Xu, Z. Xiong, M.R. Dorr, J.A. Hittinger, K. Bodi, J. Candy, B.I. Cohen, R.H. Cohen, P. Colella, G.D. Kerbel, S. Krasheninnikov, W.M. Nevins, H. Qin, T.D. Rognlien, P.B. Snyder, M.V. Umansky

September 14, 2007

Nuclear Fusion 
This document was prepared as an account of work sponsored by an agency of the United States Government. Neither the United States Government nor the University of California nor any of their employees, makes any warranty, express or implied, or assumes any legal liability or responsibility for the accuracy, completeness, or usefulness of any information, apparatus, product, or process disclosed, or represents that its use would not infringe privately owned rights. Reference herein to any specific commercial product, process, or service by trade name, trademark, manufacturer, or otherwise, does not necessarily constitute or imply its endorsement, recommendation, or favoring by the United States Government or the University of California. The views and opinions of authors expressed herein do not necessarily state or reflect those of the United States Government or the University of California, and shall not be used for advertising or product endorsement purposes. 


\title{
Edge Gyrokinetic Theory and Continuum Simulations ${ }^{1}$
}

\author{
X.Q. Xu 1), Z. Xiong 1), M. R. Dorr 1), J. A. Hittinger 1), K. Bodi 2), J. Candy 3), \\ B. I. Cohen 1), R. H. Cohen 1), P. Colella 4), G. D. Kerbel 1), S. Krasheninnikov 2), \\ W. M. Nevins 1), H. Qin 5), T. D. Rognlien 1), P. B. Snyder 3), M. V. Umansky 1) \\ 1) Lawrence Livermore National Laboratory, Livermore, CA 94551 USA \\ 2) University of California, San Diego, La Jolla, CA 92093 USA \\ 3) General Atomics, San Diego, CA 92186 USA \\ 4) Lawrence Berkeley National Laboratory, Berkeley, CA 94720 USA \\ 5) Princeton Plasma Physics Laboratory, Princeton, NJ 08543 USA \\ e-mail contact of main author: xxu@llnl.gov
}

\begin{abstract}
The following results are presented from the development and application of TEMPEST, a fully nonlinear (full-f) five dimensional $(3 \mathrm{~d} 2 \mathrm{v})$ gyrokinetic continuum edge-plasma code: (1) As a test of the interaction of collisions and parallel streaming, TEMPEST is compared with published analytic and numerical results for endloss of particles confined by combined electrostatic and magnetic wells. Good agreement is found over a wide range of collisionality, confining potential, and mirror ratio; and the required velocity space resolution is modest. (2) In a large-aspect-ratio circular geometry, excellent agreement is found for a neoclassical equilibrium with parallel ion flow in the banana regime with zero temperature gradient and radial electric field. (3) The four-dimensional $(2 \mathrm{~d} 2 \mathrm{v})$ version of the code produces the first self-consistent simulation results of collisionless damping of geodesic acoustic modes and zonal flow (Rosenbluth-Hinton residual) with Boltzmann electrons using a full-f code. The electric field is also found to agree with the standard neoclassical expression for steep density and ion temperature gradients in the banana regime. In divertor geometry, it is found that the endloss of particles and energy induces parallel flow stronger than the core neoclassical predictions in the SOL. (5) Our 5D gyrokinetic formulation yields a set of nonlinear electrostatic gyrokinetic equations that are applicable to both neoclassical and turbulence simulations.
\end{abstract}

\footnotetext{
${ }^{1}$ This work was performed under the auspices of the U.S. Department of Energy by University of California Lawrence Livermore National Laboratory under contract No. W-7405-Eng-48 at LLNL, Grant No. DE-FG02-04ER54739 at UCSD, and grants DE-FG03-95ER54309 at general Atomics and DE-AC02$76 \mathrm{CHO} 3073$ at PPPL.
} 


\section{Introduction}

Understanding the structure of the edge transport barrier in high-performance (H-mode) discharges requires a kinetic description of the plasmas because the radial width of the pedestal observed in experiments is comparable to the radial width of individual ion drift orbits (leading to a large distortion of the local distribution function from a Maxwellian), and because the ion and electron mean-free-paths are long compared to the connection length for the hot plasma at the top of the edge pedestal (violating the assumptions underlying collisional fluid models). A gyrokinetic formulation $(2 \mathrm{v})$ [1] is a reasonable approximation for edge plasmas because it is believed that pedestal physics is likely dominated by phenomena having low frequencies compared to the ion gyrofrequency. But previous gyrokinetic theories and codes do not apply to edge plasmas because they cannot treat fully nonlinear electromagnetic perturbations with multi-scale-length structures in space-time for full divertor geometry.

We report on the development and application of TEMPEST, a fully nonlinear (full-f) gyrokinetic code, to simulate H-mode edge plasmas. This 5-dimensional $\left(\psi, \theta, \zeta, E_{0}, \mu\right)$ continuum code represents velocity space via a grid in equilibrium energy $\left(E_{0}\right)$ and magnetic moment $(\mu)$ variables, and configuration space via a grid in poloidal magnetic flux $(\psi)$, poloidal angle $(\theta)$ and toroidal angle $(\zeta)$. The geometry can be circular annulus or that of a diverted tokamak and so includes boundary conditions for both closed magnetic flux surfaces and open field lines. The same set of gyrokinetic equations $[2,3]$ are discretized for both geometries. The equations are solved via a Method-of-Lines approach and an implicit backward-differencing scheme using a Newton-Krylov iteration to advance the system in time [4]. The spatial derivatives are discretized with finite differences while a high-order finite volume method is used in velocity space $\left(E_{0}, \mu\right)$. A fourth-order upwinding algorithm is used for parallel streaming, and a fifth-order WENO scheme [5] is used for particle cross-field drifts. Boundary conditions at conducting material surfaces are implemented on the plasma side of the sheath. The code includes kinetic or Boltzmann electrons. A nonlinear Fokker-Planck collision operator (CQL) from the STELLA code [6] has been extracted and integrated into TEMPEST using the same implicit Newton-Krylov solver. A new Fokker-Planck collision operator in $\left(E_{0}, \mu\right)$ space is under development for improved accuracy and conservation properties. The gyrokinetic Poisson equation is solved 
self-consistently with the gyrokinetic equations as a differential-algebraic system involving a nonlinear system solve via Newton-Krylov iteration using a multigrid preconditioned conjugate gradient (PCG) solver for the Poisson equation.

\section{Basic Gyrokinetic Equation}

A set of generalized gyrokinetic Vlasov-Maxwell equations valid for edge-plasma conditions has been derived in the gyrocenter coordinate system by the Lie transform perturbation method, which uses the Poincaré-Cartan-Einstein 1-form and the pullback transformation for the distribution function [2]. This formalism allows inclusion of nonlinear large-amplitude, time-dependent background electromagnetic fields in addition to smallamplitude, short-wavelength electromagnetic perturbations. As an example, the pullback transformation in the gyrokinetic Poisson equation is explicitly expressed in terms of moments of the gyrocenter distribution function, thus describing the important gyro-orbit squeezing effect due to the large electric field gradients in the edge and the full finite Larmor radius effect for short wavelength fluctuations. The familiar polarization-drift density in the gyrocenter Poisson equation is replaced by a more general expression.

\subsection{Fully Nonlinear Ion Gyrokinetic Equations}

The ion gyrokinetic equations presently implemented in TEMPEST for the time-dependent five-dimensional (5D) distribution functions are simplified from our recent new formulation [2] and Hahm's earlier work [3]. In order to accurately simulate particle parallel streaming, the large electrostatic potential $\Phi$, which has a multiple spatial-time scales, is split into two parts $\Phi=\Phi_{0}+\delta \phi$ : $\Phi_{0}$ is the large amplitude, slowly varying component;

$\delta \phi$ is the small amplitude, rapidly varying component. Here $E_{0}$ is defined as the total energy including $\Phi_{0}$, but not $\delta \phi$. Then $E_{0}$ is a constant of motion if $\delta \phi \sim 0$ for a coordinate aligned with the direction of phase-space flow. The kinetic equation for the gyrocenter distribution function $F_{\alpha}\left(\overline{\mathbf{x}}, \bar{\mu}, \bar{E}_{0}, t\right)$ in gyrocenter coordinates $\left(\overline{\mathbf{x}}=\mathbf{x}-\rho_{\alpha}, \rho_{\alpha}=\mathbf{b} \times\right.$ $\left.\mathbf{v} / \Omega_{\mathbf{c} \alpha}\right)$, "equilibrium energy" $\bar{E}_{0}$, and magnetic moment $\bar{\mu}$, has the form:

$$
\begin{aligned}
\frac{\partial F_{\alpha}}{\partial t}+ & \overline{\mathbf{v}}_{\mathbf{d}} \cdot \frac{\partial F_{\alpha}}{\partial \overline{\mathbf{x}}_{\perp}}+\left(\bar{v}_{\| \alpha}+v_{\text {Banos }}\right) \mathbf{b} \cdot \frac{\partial F_{\alpha}}{\partial \overline{\mathbf{x}}} \\
+ & {\left[q \frac{\partial\left\langle\Phi_{0}\right\rangle}{\partial t}+\bar{\mu} \frac{\partial B}{\partial t}-\frac{B}{B^{*}} \bar{v}_{\|} q \frac{\partial\langle\delta \phi\rangle}{\partial s}-\mathbf{v}_{\mathbf{d} \mathbf{0}} \cdot(q \bar{\nabla}\langle\delta \phi\rangle)\right] \frac{\partial F_{\alpha}}{\partial E_{0}}=C\left(F_{\alpha}, F_{\alpha}\right) }
\end{aligned}
$$




$$
\begin{aligned}
\overline{\mathbf{v}}_{\mathbf{d}} & =\frac{c \mathbf{b}}{q B_{\|}^{*}} \times(q \bar{\nabla}\langle\Phi\rangle+\bar{\mu} \bar{\nabla} B)+\bar{v}_{\|}^{2} \frac{M_{\alpha} c}{q B_{\|}^{*}}(\bar{\nabla} \times \mathbf{b}) . \\
\overline{\mathbf{v}}_{\mathbf{d} \mathbf{0}} & =\frac{c \mathbf{b}}{q B_{\|}^{*}} \times\left(q \bar{\nabla}\left\langle\Phi_{0}\right\rangle+\bar{\mu} \bar{\nabla} B\right)+\bar{v}_{\|}^{2} \frac{M_{\alpha} c}{q B_{\|}^{*}}(\bar{\nabla} \times \mathbf{b}) . \\
\bar{v}_{\|} & = \pm \sqrt{\frac{2}{M_{\alpha}}\left(E_{0}-\bar{\mu} B-q\left\langle\Phi_{0}\right\rangle\right)}, \quad v_{\text {Banos }}=\frac{\mu c}{q}(\mathbf{b} \cdot \bar{\nabla} \times \mathbf{b}), \\
B_{\| \alpha}^{*} & \equiv B\left[1+\frac{\mathbf{b}}{\Omega_{c \alpha}} \cdot\left(v_{\|} \bar{\nabla} \times \mathbf{b}\right)\right], \Omega_{c \alpha}=\frac{q B}{M_{\alpha} c}, \mu=\frac{M_{\alpha} v_{\perp}^{2}}{2 B}, \\
\langle\delta \phi\rangle & =\langle\Phi\rangle-\left\langle\Phi_{0}\right\rangle .
\end{aligned}
$$

Here $Z_{\alpha} e, M_{\alpha}$ are the electric charge and mass of electrons $(\alpha=e)$ and ions $(\alpha=i)$. The left-hand side of Eq. (1) describes particle motion in electric and magnetic fields. $C_{\alpha}$ is the Coulomb collision operator. The over-bar is used for the gyrocenter variables and \langle\rangle denotes the gyroangle averaging. Additional $\mathbf{E}_{0} \times B$ flow terms due to the large amplitude and slow variation of $\Phi_{0}$ from the complete formulation [2] will be added.

\subsection{Fully Nonlinear Gyrokinetic Poisson Equation}

The complete gyrokinetic Poisson equation has been recently derived [2], including orbit squeezing by large $E_{r}$ shearing and full FLR effect. To make it numerically tractable, two additional approximations are made here: (1) the spatial variation of the transverse $\bar{\mu}$ moments $M_{n}(\overline{\mathbf{x}})$ calculated from $F_{\alpha}\left(\overline{\mathbf{x}}, \bar{\mu}, \bar{E}_{0}, t\right)$ is assumed much slower than that of the potential in evaluating the full FLR effect; (2) the total transverse distribution function is Maxwellian with temperature $T_{\perp \alpha}$.

\subsubsection{Fully Nonlinear Gyrokinetic Poisson Equation in the Arbitrary Wave- length Regime}

In the arbitrary wavelength regime, the self-consistent electrostatic potential is computed from the gyrokinetic Poisson equation:

$$
0=-4 \pi e\left[\sum_{\alpha} Z_{\alpha} N_{\alpha}(\mathbf{x}, t)-n_{e}(\mathbf{x}, t)\right]-\sum_{\alpha} \frac{1}{\lambda_{D \alpha}^{2}}\left[\Gamma_{0}(b)-1\right] \Phi,
$$

where $\Gamma_{0}(b)=I_{0}(b) e^{-b}, b=\rho_{\alpha}^{2} \nabla_{\perp}^{2} / 2, I_{0}(b)$ is the usual zeroth-order modified Bessel function. The ion gyroradius is $\rho_{\alpha}=\sqrt{2 T_{\perp \alpha} / M_{\alpha}} / \Omega_{\alpha}$, the ion gyrofrequency is $\Omega_{\alpha}=$ $Z_{\alpha} e B / M_{\alpha} c$, and the ion Debye length is $\lambda_{D \alpha}^{2}=T_{\perp \alpha} / 4 \pi N_{\alpha} Z_{\alpha}^{2} e^{2}$. Although Eq. (7) is similar to the usual gyrokinetic Poisson equation [3], there is an important distinction. 
Our gyrokinetic Poisson equation is fully nonlinear and the gyrocenter center density $N_{\alpha}$ and perpendicular ion pressure $p_{\perp \alpha}$ are calculated from the gyrocenter distribution function $F_{\alpha}\left(\overline{\mathbf{x}}, \bar{\mu}, \bar{E}_{0}, t\right)$.

$$
\begin{aligned}
N_{\alpha}(\mathbf{x}, t) & \equiv \frac{2 \pi}{M_{\alpha}} \int B_{\|}^{*} d \bar{v}_{\|} d \bar{\mu} F_{\alpha}, & & n_{e}(\mathbf{x}, t) \equiv \frac{2 \pi}{m_{e}} \int B_{\|}^{*} d v_{\|} d \mu f_{e}, \\
p_{\perp \alpha} & =\pi B \int d v_{\|} d \bar{\mu}\left(v_{\perp}^{2} F_{\alpha}\right), & & T_{\perp \alpha}=\frac{p_{\perp \alpha}}{N_{\alpha}(\mathbf{x}, t)}
\end{aligned}
$$

Here the dot product between the density gradient vector and potential gradient vector, as well as the Debye shielding, have been dropped for simplicity in Eq. (7).

The first-order Padé approximation to $\Gamma_{0}, \Gamma_{0}-1=b /(1+b)$, is an excellent fit for $0 \leq b \leq 9$, and is therefore valid well into the typical ion gyrokinetic regime as shown previously in gyrokinetic and gyrofluid simulations $[7,8]$. Substituting a simple functional transformation $\Phi=\phi_{L}+\left[T_{\perp \alpha} /\left(N_{\alpha} Z_{\alpha}^{2} e\right)\right]\left[Z_{\alpha} N_{\alpha}(\mathbf{x}, t)-n_{e}(\mathbf{x}, t)\right]$ and the Padé approximation into Eq. (7) yields

$$
\frac{\rho_{\alpha}^{2}}{2} \nabla_{\perp}^{2} \phi_{L}=-\frac{T_{\alpha}}{N_{\alpha} Z_{\alpha}^{2} e}\left[1+\frac{\rho_{\alpha}^{2}}{2} \nabla_{\perp}^{2} \ln \left(\frac{T_{\perp \alpha}}{N_{\alpha}}\right)\right]\left[Z_{\alpha} N_{\alpha}(\mathbf{x}, t)-n_{e}(\mathbf{x}, t)\right] .
$$

where $\phi_{L}$ is calculated by the gyrokinetic Poisson solver.

\subsubsection{Fully Nonlinear Gyrokinetic Poisson Equation in the Long Wavelength Regime}

In the long wavelength limit $k_{\perp} \rho_{\alpha} \ll 1$, the self-consistent electric field is typically computed from the gyrokinetic Poisson equation for multiple species

$$
\begin{aligned}
\sum_{\alpha} \frac{\rho_{\alpha}^{2}}{2 \lambda_{D \alpha}^{2}} \nabla_{\perp} \cdot\left(\ln N_{\alpha} \nabla_{\perp} \Phi\right)+\nabla^{2} \Phi= & -4 \pi e\left[\sum_{\alpha} Z_{\alpha} N_{\alpha}(\mathbf{x}, t)-n_{e}(\mathbf{x}, t)\right] \\
& -\sum_{\alpha} \frac{\rho_{\alpha}^{2}}{2 \lambda_{D \alpha}^{2}} \frac{1}{N_{\alpha} Z_{\alpha} e} \nabla_{\perp}^{2} p_{\perp \alpha} .
\end{aligned}
$$

There are two important distinctions between Eq. (11) and the usual gyrokinetic Poisson equation [3]. Our gyrokinetic Poisson equation is fully nonlinear with the gyrocenter center density $N_{\alpha}$ and perpendicular ion pressure $p_{\perp \alpha}$ calculated from the gyrocenter distribution function $F_{\alpha}\left(\overline{\mathbf{x}}, \bar{\mu}, \bar{E}_{0}, t\right)$ defined in Eqs. (8)-(9). The last term of Eq. (11) is the diamagnetic density from the long wavelength expansion of the gyroaveraged gyrocenter density $N_{\alpha}(\mathbf{x}, t)$. Although the diamagnetic density is small compared to the ion gyrocenter density, it is of the same order as both the polarization density in high-beta plasmas 
and the difference between ion and electron gyrocenter densities! This equation is an extension of the typical neoclassical electric field model including poloidal variation [19].

\subsection{Boundary Conditions}

\subsubsection{Radial Boundary Conditions}

The radial Robin boundary conditions are used for $F_{\alpha}$ and potential $\Phi$ at the inner core surface $\psi=\psi_{c}$ and the outer wall surface $\psi=\psi_{w}$. The Robin condition is a generalization of the Dirichlet and Neumann boundary conditions. Since the gyrokinetic equation has only a first-order radial advection term, only one boundary condition is used and then only where the convection is into the domain, No boundary condition should be imposed for particles convecting out of the domain and therefore an extrapolation is used at that boundary.

\subsubsection{Poloidal Boundary Conditions}

The boundary conditions in the $\theta$ direction for $F_{\alpha}$ and for $\Phi$ are the sheath boundary conditions at the divertor plates, and a twist-shifted (in 5D) parallel periodic condition in the "core" (closed field-lines). Our present implementation for the sheath boundary condition treats a normal intersection of the flux surface with the wall, with the ions being fully absorbed and zero current through the sheath for no biasing; there can be an energetic group of impinging electrons that can escape the sheath potential and reach the wall with the energy $E_{0}+e \delta \phi_{s h}-\mu B>0$. Here $\Phi_{s h}=\Phi_{0 s h}+\delta \phi_{s h}$ is the sheath potential.

\section{i. Sheath Boundary Conditions for Potential}

If the gyrokinetic ion and fluid electron model are used, the sheath potential is determined by the ambipolarity condition:

$$
\Phi_{s h}=\frac{T_{e}}{e} \ln \left[\frac{4 \Gamma_{i, s h}}{n_{e, s h} \zeta \sqrt{8 T_{e, s h} / \pi m_{e}}}\right], \Gamma_{i, s h}=\frac{2 \pi B}{M_{\alpha}^{2}} \int_{q \Phi_{0 s h}}^{\infty} d E_{0} \int_{0}^{\left(E_{0}-q \Phi_{0 s h}\right) / B} \frac{d \mu}{\left|v_{\|}\right|} v_{\|} F_{i}^{\sigma}(.12)
$$

The $\sigma= \pm$ represents the plus and minus sheet of parallel velocity with $F_{i}^{\sigma} \neq 0$ for only incoming particle sheet. Here it is assumed that impinging electrons have a Maxwellian distribution. The factor $\zeta \equiv 1 /\left(1+\tau_{p} / \tau_{e}\right)$ includes a correction for electron long mean-free path physics. $\tau_{p}$ is long mean-free path confinement time and $\tau_{c}$ is the confinement time 
for the collisional sheath-limited case. $\zeta \equiv 1$ if the electrons are in the short mean-free path regime.

If both electrons and ions are kinetic, the sheath potential is determined by the quasineutrality condition at the sheath entrance:

$$
\begin{aligned}
\Gamma_{i, s h} & =\frac{2 \pi B}{M_{\alpha}^{2}} \int_{q \Phi_{0 s h}}^{\infty} d E_{0} \int_{0}^{\left(E_{0}-q \Phi_{0 s h}\right) / B} \frac{d \mu}{\left|v_{\|}\right|} v_{\|} F_{i}^{\sigma}, \\
\Gamma_{e, s h} & =\frac{2 \pi B}{m_{e}^{2}} \int_{e \delta \phi_{s h}}^{\infty} d E_{0} \int_{0}^{\left(E_{0}-e \delta \phi_{s h}\right) / B} \frac{d \mu}{\left|v_{\|}\right|} v_{\|} F_{e}^{\sigma}, \quad \Gamma_{i, s h}=\Gamma_{e, s h} .
\end{aligned}
$$

\section{ii. Sheath Boundary Conditions for Distribution Functions}

If the gyrokinetic ion and fluid electron model is used, the ion distribution function is:

$$
F_{\alpha}\left(\psi, \theta, E_{0}, \mu\right)=\left\{\begin{array}{cc}
F_{\alpha}\left(\psi, \theta, E_{0}, \mu\right), & v_{\|} \geq 0 \\
0, & v_{\|} \leq 0
\end{array}\right.
$$

A convention regarding the sign of the parallel velocity is that it is positive when there is a positive projection on the $\theta$ axis. Here the positive $\theta$ axis is pointing to the plate/wall.

If both electrons and ions are kinetic, the electron distribution function is:

$$
f_{e}\left(\psi, \theta, E_{0}, \mu, \sigma=-1\right)=\left\{\begin{array}{cc}
f_{e}\left(\psi, \theta, E_{0}, \mu, \sigma=1\right), & \left|v_{\|}\right| \leq v_{S H} \\
0, & \left|v_{\|}\right| \geq v_{S H}
\end{array}\right.
$$

Here $v_{s h}=\sqrt{2 e \Phi_{s h} / m_{e}}$ is the electron threshold velocity determined by the sheath potential $\Phi_{s h}$.

\section{TEMPEST Simulation Schemes}

The TEMPEST gyrokinetic equations and gyrokinetic Poisson (GKP) equation are selfconsistently integrated as a differential-algebraic system involving a nonlinear system solve via Newton-Krylov iteration. The spatial derivatives are discretized with finite differences while a high-order finite volume method is used in velocity space $\left(E_{0}, \mu\right)$. A fourth-order upwinding algorithm is used for parallel streaming, and a fifth-order WENO scheme [5] is used for particle cross-field drifts. The GKP equation, the drift velocities and acceleration are discretized using centered differencing. Boundary conditions at conducting material surfaces are implemented on the plasma side of the sheath. The GKP preconditioner block is inverted using a multigrid preconditioned conjugate gradient (PCG) 
solver. The PCG solver and preconditioners are provided by the Hypre library using the "semi-structured interface"[9]. The code includes kinetic or Boltzmann electrons. The Boltzmann relation in the adiabatic option employs flux surface averaging to maintain neutrality within field lines and is solved self-consistently with the GKP equation. A decomposition procedure circumvents the near singularity of the GKP Jacobian block that otherwise degrades CG convergence. A nonlinear Fokker-Planck collision operator (CQL) from the STELLA code [6] has been extracted and integrated into TEMPEST using the same implicit Newton-Krylov solver. A new Fokker-Planck collision operator in $\left(E_{0}, \mu\right)$ space is under development for improved accuracy and conservation properties [10].

Tempest uses a Python scripting front end that allows the gyrokinetic code to interface with other codes, such as the edge transport code UEDGE and other physics packages, implemented as Python modules. We have designed and implemented flexible $\mathrm{C}++$ data structures for the management of distributed arrays and supporting data objects on top of SAMRAI (Structured Adaptive Mesh Refinement Application Infrastructure) [11]. The data layout is defined on distributed and disjoint unions of rectangular blocks, but with arbitrary interblock connectivity (nonlocal communication) for multiple regions in the edge plasma across the magnetic separatrix.

The gyrokinetic equation is primarily particle convection both in configuration and velocity spaces. An accurate and stable convection scheme is extremely important for the success of continuum gyrokinetic simulations. Here we present tests of our convection scheme for parallel streaming and toroidal drift. The fourth-order upwind scheme for parallel streaming is given in the appendix. The complete description and tests of the code algorithms will be given in a future publication.

\subsection{Parallel streaming}

The streaming test is done in a circular geometry with the magnetic field $B(\theta)=B_{0}(1-$ $\epsilon \cos \theta) \epsilon=0.3, r=0.51, B_{p}=0.3$. The range of the variables: $0 \leq \theta \leq 2 \pi, 0 \leq v_{\|} \leq$ $3 v_{t h}$. The initial distribution function is $F_{0}=\left(B_{0} / B\right) F_{M}$, where $F_{M}$ is a Maxwellian distribution. For a given energy $E_{0}$ and magnetic moment $\mu$ in velocity space, $F_{0}$ has a pulse profile in $\theta$-coordinate due to $B(\theta)$. As the time evolves, the pulse will propagate along the magnetic field due to the parallel streaming. The system evolves toward a 

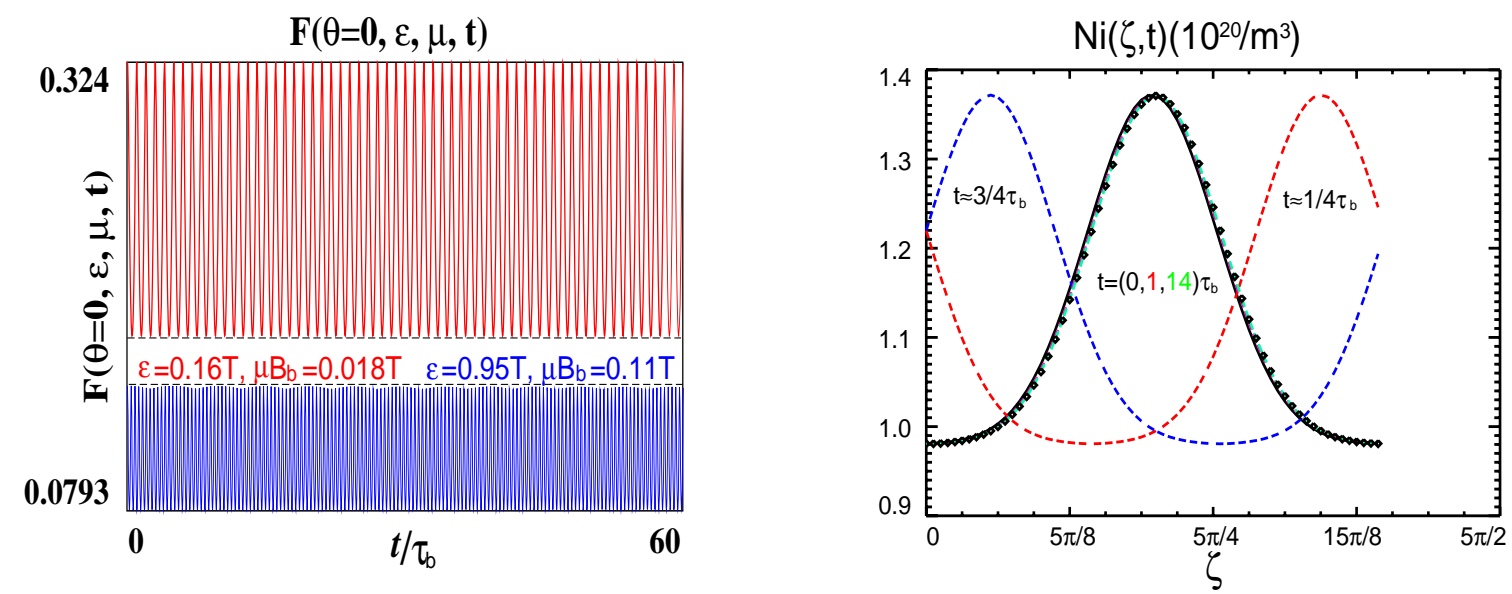

FIG. 1: (a). Time evolution of the distribution function for circulating particles using a fourthorder upwinding scheme. $\nu^{*}=0.0, \phi_{0}=0.0, \epsilon=0.3, h=0.51, Z_{\alpha}=1, B_{p}=0.3$. (b). The density vs toroidal angle at different times for toroidal convection using a 5th-order Weno scheme.

quasi-equilibrium. The oscillations at a specified poloidal position $\theta$ for a given energy $E_{0}$ and $\mu$ in velocity space represent the convection along the particle trajectory for a closed magnetic field line. For an ideal numerical difference scheme for convection, the amplitude should exhibit regular oscillations in time around a constant mean. The time evolution of the distribution function is plotted in Fig. 1(a), which shows an almost ideal oscillation for about the expected 60 cycles of a circulating particle far away from the trappinguntrapping boundary using the forth-order upwinding scheme given in the appendix. For a second-order upwinding scheme, a slight downward drift is observed; while for the third-order upwinding scheme, a slightly damping is observed. However, even with the fourth-order upwinding scheme, the damping is strong for barely circulating particles and barely trapped particles due to non-uniform (orbit time $\tau$ ) grid spacing (not shown).

\subsection{Toroidal convection}

A similar test has been done for toroidal drift using a 5th-order Weno scheme. Fig. 1(b) shows the density vs toroidal angle for different times. An initial pulse is prescribed, centered at the middle of toroidal simulation domain. As the time evolves, the pulse propagates due to toroidal drift and should come back to its initial position with the same shape due to toroidal periodicity. A good numerical scheme should preserve the property. As we can see from the Fig. 1(b), our scheme preserves the property very well 
after the 14 cycles. There is no significant damping or deformation from the original pulse. The primary reason for using the 5th-order Weno scheme is the exisitence of radial regions for particles with given energy $E_{0}$ and magnetic moment $\mu$ that are inaccessible under the influence of radial magnetic drifts.

\section{TEMPEST Simulation Results}
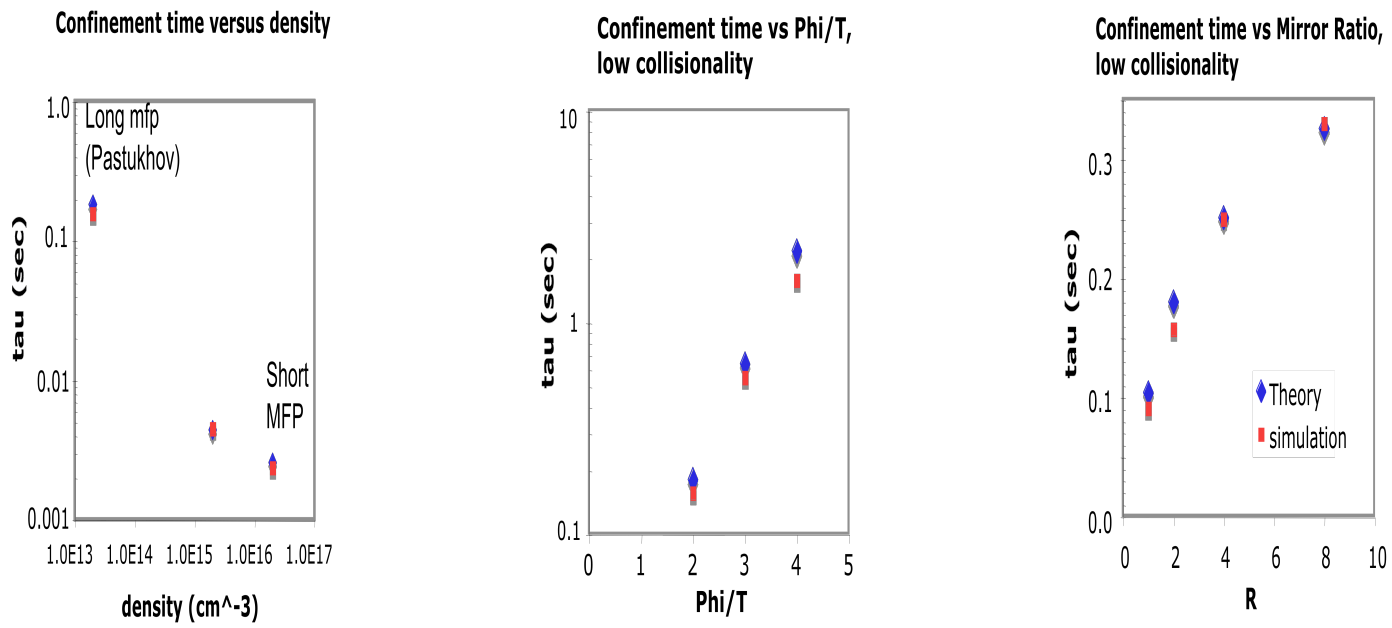

a)

b)

c)

FIG. 2: Collisional endloss ("Pastukhov") test cases: (a) confinement time versus density; (b) confinement time versus potential e $\phi / T_{e}$ at low collisionality; (c) confinement versus mirror ratio at low collisionality.

To facilitate verification and validation, both full-f and delta-f options are available for either circular or divertor geometry. TEMPEST is runnable as (1) 3D for parallel streaming and the scrape-off-layer (SOL) physics with endloss $F\left(\theta, E_{0}, \mu\right)$; $(2) 4 \mathrm{D}$ for axisymmetric transport $F\left(\psi, \theta, E_{0}, \mu\right)$, and (3) $5 \mathrm{D}$ for turbulence $F\left(\psi, \theta, \zeta, E_{0}, \mu\right)$. The different aspects of 3D, 4D and 5D TEMPEST have been verified on various known physics problems: (1) collisional scattering into a velocity-space loss cone; (2) neoclassical flow and transport; (3) electric field generation and geodesic acoustic mode damping; and (4) selfconsistent radial electric field for steep density and ion temperature gradients; (5) drift waves and ion temperature gradient (ITG) modes. 


\subsection{D Pastukhov Collisional Endloss}

As a test of collisional velocity-space transport and parallel streaming, 3D TEMPEST $(1 \mathrm{~d} 2 \mathrm{v})$ simulation results are compared with published analytical and numerical results as shown in Fig. 2 for the endloss of particles confined by combined electrostatic and magnetic wells $[12,13,14]$. Here the electrostatic and magnetic field are uniform in simulation volume, with abrupt abrupt increases at the walls (incorporated into the boundary conditions).Good agreement is found over a wide range of collisionality, confining potential, and mirror ratio; the required velocity-space resolution is modest. In these simulations, the linearized CQL collision package is used.
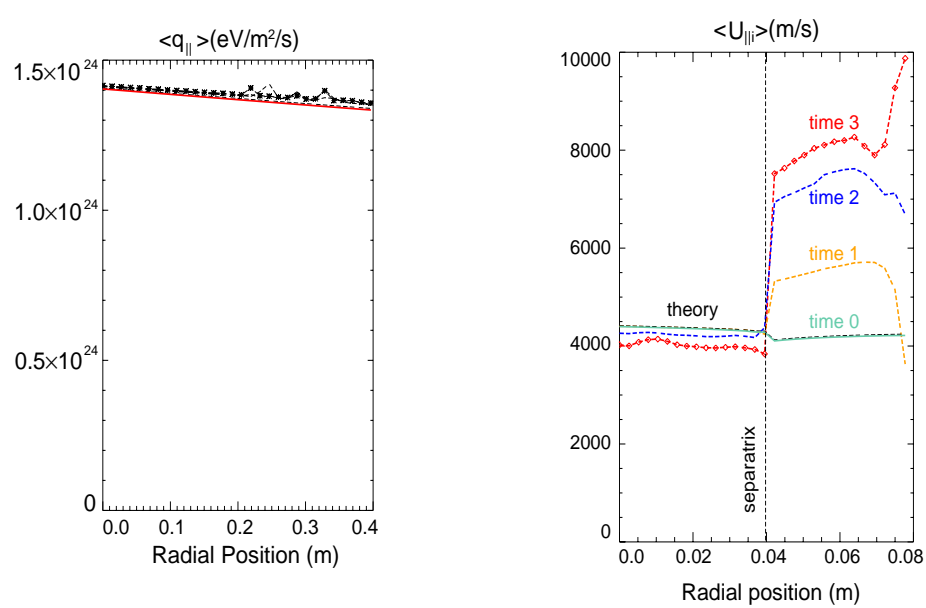

a)

b)

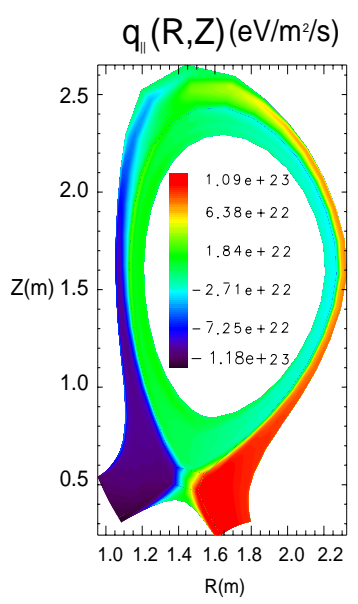

c)

FIG. 3: (a) Comparison between simulation results with theory for a collisionless case with $\nabla T_{i}=0$ and zero finite banana orbit width. Flux surface averaged parallel heat flux $\left\langle q_{\| i}\right\rangle$. The solid lines are theory and the other lines are for different times; (b) Comparison of simulation results with theory for flux surface averaged parallel flow velocity $\left\langle U_{\| i}\right\rangle$ in the banana regime with $\nu_{* i} \simeq 0.02, \nabla T_{i}=0$ and finite banana orbit width in X-point divertor geometry. The average is done by integration along the field-line from the inner plate to outer plate in the SOL. (c) The contours of parallel heat flux $q_{\|}(R, Z)$ in the divertor geometry.

\subsection{D Neoclassical flows}

For a shifted Maxwellian distribution that analytically satisfies Eq. (1) as an initial condition, TEMPEST should preserve the solution without any significant change (within 
our finite-difference truncation accuracy) after running some time steps. We tested such a case using the following simulation parameters: inverse aspect ratio $\epsilon=a / R_{0}=0.03$, the major radius $R_{0}=17.1$ meter, toroidal magnetic field $B_{t}=1.5 T$, and poloidal magnetic field $B_{p}=0.2 T$. The ion density and temperature profiles used are $n_{i}(\psi)=$ $N_{i x} \exp \left(-\ln \left(N_{i x} / N_{i o}\right) \psi / L_{\psi}\right), T_{i}(\psi)=T_{i x} \exp \left(-\ln \left(T_{i x} / T_{i o}\right) \psi / L_{\psi}\right)$ with $T_{i x}=3 k e V, T_{i o}=$ $0.95 T_{i x}, N_{i x}=1 \times 10^{20} \mathrm{~m}^{-3}$, and $N_{i o}=0.95 N_{i x}$. The mesh resolutions are $n_{\psi}=30, n_{\theta}=$ $50, n_{E}=60$, and $n_{\mu}=30$. In the simulations $\Phi$ is set to zero for simplicity. As shown in Fig. 3(a), the simulation results remain in good agreement with theoretical prediction even after 10000 time steps ( 50 thermal ion transit times). The solid line in plot of $q_{\| i}$ comes from theoretical prediction for a shifted Maxwellian distribution, $q_{\| \alpha}=2.5 N_{\alpha} U_{\| \alpha} T_{\alpha}$, and $U_{\| \alpha}=-\left(I / \Omega_{\alpha}\right)\left(T_{\alpha} / M_{\alpha}\right)\left(\partial \ln N_{\alpha} / \partial \psi\right)$.

In divertor geometry with given particle and heat sources on the inner core boundary surface, it is found that the dominance of rapid parallel endloss of particles and energy in the SOL induces a parallel flow that is stronger than core neoclassical predictions in the SOL as indicated in Fig. 3(b); a symmetry point is developed for the parallel heat flux on the top of the machine as expected as shown in Fig. 3(c).

\subsection{D Geodesic-Acoustic Modes}

The Geodesic-Acoustic Mode (GAM) is an asymmetric mode, which involves parallel ion dynamics, cross-field drifts, and acceleration. Earlier GAM theory and simulations focused on the large aspect ratio, small orbit $[15,16]$ regime. Recently Sugama and Watanabe found that the damping rate is sensitive to $k_{\perp} \rho_{i}$ at large $q$ due to the effect of large banana orbits [17]. In our 4D GAM simulations, the charge is radially separated by an initial sinusoidal perturbation of the ion density. The electron model is Boltzmann $n_{e}=$ $\left\langle n_{i}(\psi, \theta, t=0)\right\rangle \exp \left(e \phi / T_{e}\right) /\left\langle\exp \left(e \phi / T_{e}\right)\right\rangle$, where \langle\rangle represents the flux surface average. This choice of coefficient for Boltzmann electron model means that there is no cross field electron transport. Both radial and poloidal boundary conditions are periodic. The first full-f, self-consistent simulation results of collisionless damping of geodesic acoustic modes and zonal flow are plotted in Fig. 4. Good agreement is shown between theory $[17,18]$ and simulations for the frequency of GAMs in Fig. 4(a) and damping rate in Fig. 4(b). The $30 \%$ difference between theory and simulation may be due to the theory using an 
asymptotic $1 / q^{2}$ expansion for large $\mathrm{q}$, while $\mathrm{q}=2.2$ in the simulation is not very large. The large effect of the orbit size on the GAM damping rate is illustrated in Fig. 4(c). For the same parameters, the damping rate is almost zero if the finite banana orbit effect is ignored.
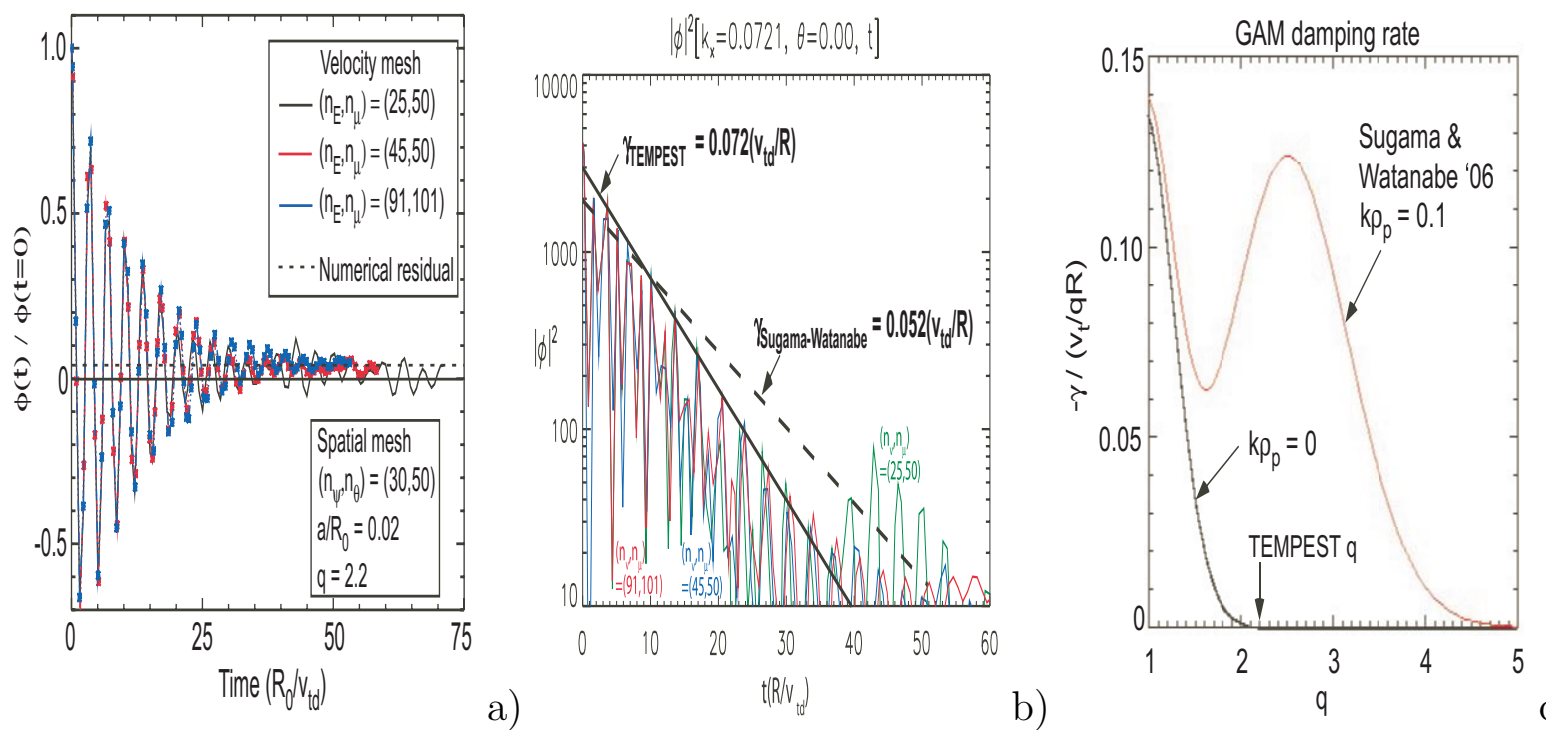

FIG. 4: (a) Time evolution of the zonal-GAM potential shows GAM oscillation, collisionless damping, and residual for a large-aspect-ratio circular geometry with $q=2.2$ and $\epsilon=0.02$ with three different velocity-space resolutions; (b) Comparison of simulation results with theory for the GAM damping rate at three different velocity resolutions in finite banana orbit regime; (c) GAM damping rate vs $q$ with finite banana orbit effect (red) and without finite banana orbit effect (black) from Sugama and Watanabe theory [17].

\subsection{D Neoclassical Radial Electric Field with Finite Orbits}

The simulations presented here are carried out for large aspect ratio circular geometry with the magnetic field $B_{t}=15 T, R_{0}=17.1 \mathrm{~m}, q=2.3$ and $\epsilon=0.02$. The ion density and temperature profiles are initialized as a tanh function of radius centered around the middle of simulation domain $\left[N(x)=n_{0}+n_{m} \tanh \left(\left(x-x_{m}\right) / \Delta_{n}\right)\right]$. The boundary ion distribution is a fixed Maxwellian with $N\left(x_{0}\right)=1 \times 10^{20} \mathrm{~m}^{-3}, N\left(x_{L}\right)=5 \times 10^{19} \mathrm{~m}^{-3}, T_{i}\left(x_{0}\right)=3 \mathrm{keV}$, and $T_{i}\left(x_{L}\right)=1.5 \mathrm{keV}$ during a simulation. The radial boundary condition for the potential is $\phi\left(x_{0}\right)=\phi\left(x_{L}\right)=0$. There are two radial boundary buffer zones where the ion distribution function is forced toward the initial Maxwellian by a Krook-type collision term with large damping rate at the boundaries and negligibly small rate in the center region to avoid 
complications associated with banana orbit intersections at the boundaries. The electron model is the Boltzmann model specified in section 3.3. The neoclassical radial electric field from TEMPEST simulations agrees very well with the standard neoclassical expression $\left\langle U_{i \|}\right\rangle=\left(c T_{i} / Z_{i} e B_{p}\right)\left[k\left(\partial \ln T_{i} / \partial r\right)-\left(\partial \ln P_{i} / \partial r\right)-\left(Z_{i} e / T_{i}\right)(\partial\langle\Phi\rangle / \partial r)\right]$ with $k=1.17$ as shown in Fig. 5(a), even in a region where the density gradient scale length is comparable to the banana orbit size[19]. The radial electric field is generated due to the neoclassical polarization and the relative maximum charge separation is only $0.4 \%$ as shown in Fig. 5(b). A time history of the flux surface averaged electric potential in Fig. 5(c) shows geodesic acoustic oscillations generated by the initial conditions, which then relax to a near steady state, consistent with the previous studies[19, 20]. Due to the steep ion density and temperature gradient, $E_{r}$ is much larger than the Rosenbluth-Hinton residual as discussed in the previous section.
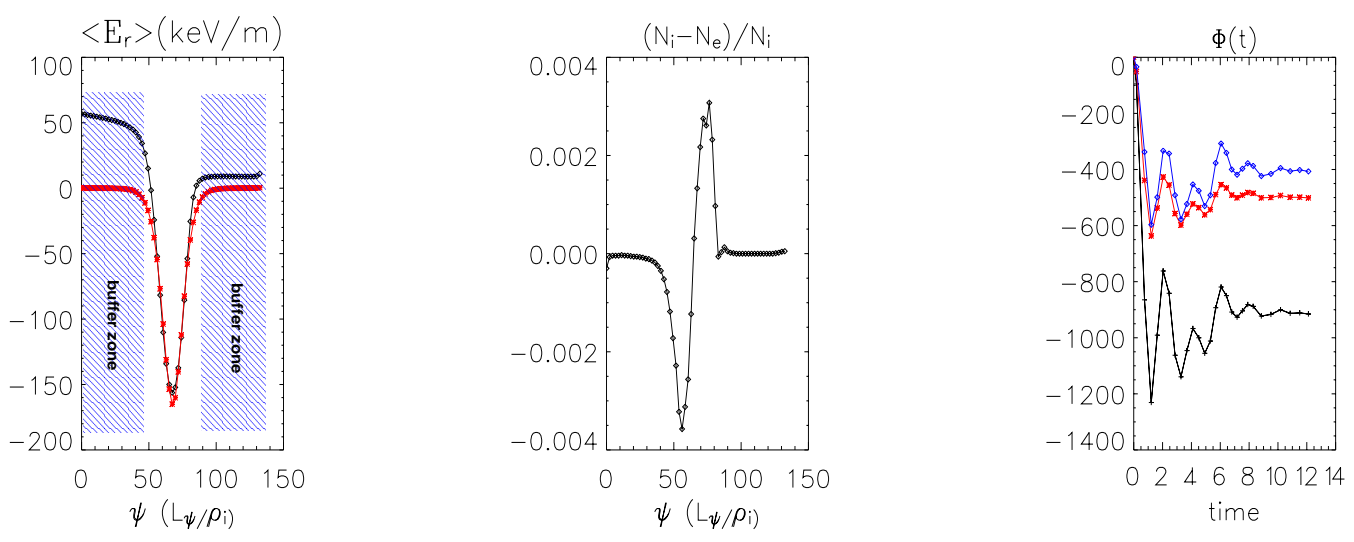

a)

b)

c)

FIG. 5: (a) E from TEMPEST simulations (black) vs neoclassical theory (red) with finite banana orbit effect in circular geometry with $q=2.3$ and $\epsilon=0.02$; (b) the charge separation due to finite ion banana orbit effect; (c) time evolution of the flux surface averaged electrostatic potential at different radial locations.

\subsection{D Drift Waves and ITG Modes}

Conventional orderings are different for 4D neoclassical transport and 5D $\left(\psi, \theta, \zeta, E_{0}, \mu\right)$ turbulence where $\zeta$ as the binormal coordinate. Our 5D gyrokinetic formulation yields a set of nonlinear electrostatic gyrokinetic equations that are valid for both neoclassical and 
turbulence simulations. In particular, the field solver for shear/zonal flow is different from that for turbulence due to: (1) the strong poloidal variation of the electrostatic potential in the divertor X-point geometry originating from different boundary conditions in the core, the SOL, and private-flux regions; (2) additional terms are promoted by the edge ordering of a large background $\mathbf{E} \times \mathbf{B}$ flow $u_{E} \simeq v_{T i} .5 \mathrm{D}$ tempest uses field-aligned coordinates with 4th-order interpolation and $\zeta$-index shifting for twist-shifted parallel boundary condition in the core inside the separatrix and for radial differencing in the usual flux coordinates to minimize the cell distortion due to magnetic shear. 5D TEMPEST shows good agreement with theory for the drift-wave frequency with $10 \%$ radial variation of the ion density and a flat ion temperature profile. For a different simulation setup with $10 \%$ radial variation of ion temperature profile and the flat ion density profile, 5D Tempest also shows an ITG mode. Benchmarks with theory and other codes are in progress.

\section{Summary and Conclusions}

The recently developed full-f, 5D continuum edge-plasma code TEMPEST utilizes highorder spatial differencing and a high-order finite-volume scheme for velocity space to accurately simulate particle convection and Coulomb collisions. TEMPEST runs in both full divertor geometry for maximum applicability to diverted tokamaks and in circular geometry for benchmarking with analytic theories and existing core gyrokinetic codes. TEMPEST demonstrates expected physics results in 3D, 4D and 5D verification tests. The further improvement and development of TEMPEST will yield a valuable predictive model for the edge pedestal. This work is focused on a fundamental understanding of relevant physics from first-principles theory and simulations and should greatly increase our confidence in predictions of ITER edge-plasma performance.

\section{Acknowledgments}

Authors wishing to acknowledge assistance or encouragement from Drs. J. D. Lindl and W. A. Lokke. We thank Drs. Alain J. Brizard, L. Chen, A. Dimits, W. D Dorland, T. S. Hahm, G. Hammet, W. X. Wang, and T. -H. Watanabe for fruitful physics discussions. 


\section{Appendix: Fourth-order upwind scheme for non-uniform grid spacing}

We write the finite difference approximation to the first derivative at node $i$ for a discretization of $\mathrm{N}$ points as

$$
\left(\partial_{\tau} f\right)_{i} \approx \sum_{j=1}^{N} a_{j}(i) f_{j} .
$$

The function value at $i$ is written $f_{i}=f\left(\tau_{i}\right)$, where $\tau_{i}$ is defined in Eq. (44). For a fourthorder upwind-biased stencil at node $i$, the support of $\left\{\tau_{i-3}, \tau_{i-2}, \tau_{i-1}, \tau_{i}, \tau_{i+1}\right\}$ leads to the following weights:

$$
\begin{aligned}
a_{i+1}(i) & =\frac{\left(\tau_{i}-\tau_{i-1}\right)\left(\tau_{i}-\tau_{i-2}\right)\left(\tau_{i}-\tau_{i-3}\right)}{\left(\tau_{i+1}-\tau_{i}\right)\left(\tau_{i+1}-\tau_{i-1}\right)\left(\tau_{i+1}-\tau_{i-2}\right)\left(\tau_{i+1}-\tau_{i-3}\right)}, \\
a_{i}(i)= & \frac{1}{\left(\tau_{i}-\tau_{i-1}\right)}+\frac{1}{\left(\tau_{i}-\tau_{i-2}\right)}+\frac{1}{\left(\tau_{i}-\tau_{i-3}\right)}-\frac{1}{\left(\tau_{i+1}-\tau_{i}\right)}, \\
a_{i-1}(i) & =-\frac{\left(\tau_{i+1}-\tau_{i}\right)\left(\tau_{i}-\tau_{i-2}\right)\left(\tau_{i}-\tau_{i-3}\right)}{\left(\tau_{i+1}-\tau_{i-1}\right)\left(\tau_{i}-\tau_{i-1}\right)\left(\tau_{i-1}-\tau_{i-2}\right)\left(\tau_{i-1}-\tau_{i-3}\right)}, \\
a_{i-2}(i) & =\frac{\left(\tau_{i+1}-\tau_{i}\right)\left(\tau_{i}-\tau_{i-1}\right)\left(\tau_{i}-\tau_{i-3}\right)}{\left(\tau_{i+1}-\tau_{i-2}\right)\left(\tau_{i}-\tau_{i-2}\right)\left(\tau_{i-1}-\tau_{i-2}\right)\left(\tau_{i-2}-\tau_{i-3}\right)} \\
a_{i-3}(i) & =-\frac{\left(\tau_{i+1}-\tau_{i}\right)\left(\tau_{i}-\tau_{i-1}\right)\left(\tau_{i}-\tau_{i-2}\right)}{\left(\tau_{i+1}-\tau_{i-3}\right)\left(\tau_{i}-\tau_{i-3}\right)\left(\tau_{i-1}-\tau_{i-3}\right)\left(\tau_{i-2}-\tau_{i-3}\right)}
\end{aligned}
$$

with $a_{j}(i) \equiv 0$ otherwise. The formal leading-order truncation error is

$$
-\frac{\left(\tau_{i+1}-\tau_{i}\right)\left(\tau_{i}-\tau_{i-1}\right)\left(\tau_{i}-\tau_{i-2}\right)\left(\tau_{i}-\tau_{i-3}\right)}{120}\left(\partial_{\tau}^{5} f\right)_{i} .
$$

\section{References}

[1] Brizard A J and Hahm T S, 2007 submitted to Rev. Mod. Phys..

[2] Qin H, Cohen R H, Nevins W M, and Xu X Q, (2006) Contrib. Plasma Phys., 46, 477

[3] Hahm T S, 1996 Phys. Plasmas 3, 4658

[4] Hindmarsh A C, Brown P N, Grant K E, Lee S L, Serban R, Shumaker D E, and Woodward C S, 2005 ACM Transactions on Mathematical Software, 31(3), pp. 363396.

[5] Jiang G S and Shu C W, 1996 J. Comput. Phys. 126, 202

[6] Kupfer K, Harvey R W, Sauter O, et al., 1996 Phys. Plasmas 3, 3644

[7] Dorland W and Hammett G W, 1993 Phys. Fluids B 5, 812.

[8] Dimits A, 1992 IAEA technical committee meeting. 
[9] Falgout R D, Jones J E, and Yang U M, 2006 "The Design and Implementation of hypre, a Library of Parallel High Performance Preconditioners", chapter in Numerical Solution of Partial Differential Equations on Parallel Computers, A.M. Bruaset and A. Tveito, eds., Springer-Verlag, 51, pp. 267-294.

[10] Xiong Z, Cohen R H, Rognlien T D, Xu X Q, 2007 submitted to J. Comput. Phys..

[11] Hornung R D and Kohn S R, 2002 Concurrency and Computation: Practice and Experience, 14, 347-368.

[12] Rognlien, T D and Cutler, 1980 Nuclear Fusion 20, 1003.

[13] Cohen R H, 1979 Nuclear Fusion 19, 1295.

[14] Najmabadi, et al., 1994 Nuclear Fusion 24, 75.

[15] Rosenbluth, M N and Hinton F L, 1998 Phys. Rev. Lett., 80, 724

[16] Lebedev V B, Yushmanov P N, and Diamond P H, 1996 et al. Phys. Plasmas 3, 3023

[17] Sugama H and Watanable T H, 2006 J. Phys. Plasmas,72, 825-828.

[18] Zhe Gao, K. Itoh, H. Sanuki, and J. .Q. Dong, 2006 FEC2006, TH/P2-5.

[19] Wang W X and Hinton F L,2001 Phys. Rev. Lett., 80 055002-1.

[20] Novakovskii S V, et al., 1997 Phys. Plasmas 4, 4272. 